\title{
Crisis financiera global y su impacto en la dinámica bursátil europea y americana
}

\author{
Dra. Miriam Sosa \\ Facultad de Economía. Universidad Nacional Autónoma de México \\ Dr. Edgar Ortiz \\ Profesor-Investigador, Programa de Posgrado en Ciencias Políticas y Sociales. Universidad \\ Nacional Autónoma de México \\ Dra. Alejandra Cabello \\ Profesora- Investigadora, Facultad de Química. Universidad Nacional Autónoma de \\ México ${ }^{1}$ \\ (Recibido 13 de Octubre 2017, aceptado 09 de Enero 2017.)
}

\section{Resumen}

El objetivo principal de la presente investigación es analizar el impacto de la crisis financiera global en la dinámica de los mercados accionarios más importantes delos continentes americano y europeo. Para lograr dicho objetivo, se modela la volatilidad a partir de modelos GARCH simétricos y asimétricos con dummy en la ecuación de la varianza.El periodo de estudio incluye series diarias del primero de enero del 2003 al 27 de febrero del año 2015. Los resultados sugieren que la crisis financiera global impactó el comportamiento de las bolsas de valores, incrementando su volatilidad y la asimetría en la misma, sobre todo en el caso de las bolsas europeas; dichos resultados tienen importantes implicaciones para la administración del riesgo y construcción de portafolios que involucran activos de los mercados europeos y americanos. La originalidad del trabajo subyace en el análisis del impacto de la crisis financiera global, tema sumamente importante, a partir de una metodología que no se había empleado anteriormente para analizar el comportamiento de las bolsas de valores bajo estudio.

Clasificación JEL: G10, G01, C58.

Palabras clave: Volatilidad asimétrica, GARCH, TARCH, América Latina, Crisis financiera.

\section{Global Financial Crisis and its Impact on European and American Stock Markets \\ Behavior}

\begin{abstract}
This paperaims to analyze the impact of global financial crisis on the behavior of the most important European and American stock markets.Symmetric and asymmetric GARCH models with a dummy in the variance equation are employed to model stock market's volatility. Results confirm the crisis impact on the stock indices behavior; increasing volatility and raising the leverage effect, above all in the European markets. The sample period includes daily observations from January 1, 2003 to February 27, 2015.The
\end{abstract}

\footnotetext{
${ }^{1}$ Apartado 21-204. Col Coyoacán., Del Coyoacán. 04000 México, D.F. Tel: (5525) 565819
} 49. Fax: (5525) 565819 49. edgaro@unam.mx 
empirical evidence suggests that the global financial crisis impacted the behavior of the stock markets under analysis increasing their volatility and asymmetry, particularly in the case of the European markets. These findings have important implications on risk hedging and portfolio construction concerning assets from European and American share markets.Originality of this paper consists in analyzing the impact of the global financial crisis, the stock market behavior of the countries under study, applying a methodology previouslynot employed.

JEL Classification: G10, G01, C58.

Key Words: Asymmetric Volatility, GARCH, TARCH, Latin America, Financial Crisis.

\section{1.- Introducción}

La crisis financiera global ha sido uno de los fenómenos económicos y financieros más trascendentales del siglo XXI, debido a su rápida transmisión y a que su magnitud, solamente, es comparablela crisis de 1929. Este importante acontecimiento tuvo su origen en agosto de 2007, a partir de los desequilibrios generados en el mercado hipotecario estadounidense con la denominada crisis subprime. Dada la importancia de la economía estadounidense y su participación como origen y destino de inversión de cartera, los efectos en su mercado de valores se transmitieron, casi de manera inmediata, hacia otros mercados de capital, afectando, tanto en el corto como en el largo plazo, su funcionamiento.

La crisis subprime alcanzó dimensiones globales a partir de octubre del año 2008, con efectos inmediatos hasta el año 2012 y remotos o secundarios hasta el año 2015, dando lugar a importantes desequilibrios, sobre todo en países desarrollados, los cuales se transmitieron y generaron crisis a nivel mundial en diversas regiones, dentro de las que destaca la Unión Europea con la crisis de la deuda soberana.Más allá de la delimitación de diversos acontecimientos y fenómenos financieros, la crisis financiera global es vista como un periodo de inestabilidad financiera, pero sobretodo bursátil, en el que se han presentado desequilibrios en diferentes regiones a nivel global con efectos paralelos y simultáneos difíciles de diferenciar entre sí, los cuales han sido transmitidos a través de shocks entre diversos mercados de capital.

En relación al periodo de estudio, de acuerdo con Mishkin (2010) la crisis subprime tuvo efectos acotados en el sistema hipotecario estadounidense de agosto del año 2007 a agosto del año 2008, volviéndose de carácter global a partir de la caída de LehmanBrothers el 15 
de septiembre del año 2008. Contribuyendo a la generación de desequilibrios regionales, entre los que destaca la crisis de la deuda soberana la cual inició a finales del año 2008, que tuvo importantes efectos bursátiles de octubre del 2009 a diciembre del 2011 (Lee, Aizenman, Jinjarak y Park, 2012). Sin embargo, autores como Laeven y Valencia (2012) apuntan a que tuvo efectos inmediatos en un periodo mayor, en la primera mitad del año 2012 con la restructuración de la deuda pública de Grecia, e incluso, hasta el año 2015 con debates sobre la salida de dicho país del euro área (Zestos, 2015). No obstante y a pesar de los esfuerzos que se han realizado por delimitar los periodos de ocurrencia de los fenómenos financieros (crisis subprime y de la deuda soberana), sus efectos y transmisión por medio del canal bursátil se han presentado de manera paralela y en ocasiones simultánea, por la persistencia de algunos de estos shocks. Es por ello que, el periodo en el que se analizan los efectos de la crisis financiera global comprende de 9 agosto del año 2007 al 27 de febrero del 2015.

Las inestabilidades financieras han constituido un fenómeno que, de manera creciente, ha azotado a numerosos países y regiones del mundo, incrementando cada vez más el número de agentes implicados, debido a la profundización en los mecanismos de transmisión de crisis de un país a otro, presentándose los efectos en zonas geográficas muy lejanas al país donde inicialmente se inició el desequilibrio. Este fenómeno se encuentra inmerso en un proceso de integración y liberalización financiera, en el cual los mercados de valores han jugado un papel sumamente importante, haciendo posible la transmisión de desequilibrios a través de la actividad bursátil internacional.Así, la importancia del mercado de valores radica en su participación, no solo como espacio de interacción entre agentes que reciben y otorgan financiamiento, sino también como canal de transmisión de desequilibrios bursátiles a nivel global.

Con base en lo anterior, el presente artículo analiza la naturaleza de la volatilidad accionaria, de los principales mercados europeos y americanos, así como los cambios que dicha volatilidad experimentó a partir de los efectos de la crisis financiera global.El trabajo se encuentra estructurado de la siguiente manera: en la sección 2 se expone la literatura relacionada que ha sido desarrollada recientemente; la tercera sección describe la 
metodología y variables seleccionadas; la sección 4 presenta los resultados de la aplicación del modelo y su análisis; por último, la quinta sección concluye el trabajo.

\section{Estudios relacionados}

A medida que las crisis internacionales han sido más profundas, extensas y frecuentes, el reto de los investigadores y científicos vinculados con este tema ha sido cada vez mayor, dando lugar al desarrollo de distintas técnicas y metodologías, cada vez más refinadas y capaces de capturar mejor las características de las series. Lo anterior ha permitido obtener mayor información sobre las relaciones existentes entre ciertos mercados en un periodo de tiempo determinado, aportando información para los distintos actores, tal es el caso de gobiernos, instituciones financieras, empresas emisoras y público en general con cierta participación en las bolsas de valores (pensionados y participantes en fondos de inversión).

Los modelos GARCH han sido ampliamente utilizados para analizar el comportamiento de las series económicas y financieras debido a su capacidad de capturar las características de las series financieras, también denominadas como hechos estilizados, dentro de las que se encuentran: asimetría, leptocurtósis (colas pesadas y apuntamiento), conjuntos y persistenciaen la volatilidad, memoria larga y correlaciones en los cuadrados.

Gracias a las bondades de los modelos GARCH, extensa literatura empírica ha sido desarrollada en torno a: el análisis de la volatilidad de precios de mercancías (Basher y Sadorsky, 2016; Chan y Grant 2016; Hachula yHoffmann, 2015), la estimación de precios de productos financieros derivados (Teterin, Brooks y Enders, 2016; Badescu, Cui y Ortega, 2015; Pilbeam y Langeland, 2015), la realización de pronósticos en diversos mercados (Klein y Walther, 2015; Huh y Seong, 2015; Kourtis, Markellos y Symeonidis, 2016) y,entre muchas otras aplicaciones, el estudio del impacto de las crisisen la volatilidad de los precios (Apergis, 2015; Babalos, Caporale y Spagnolo, 2016; Kim, Kim y Lee, 2015).

De manera particular, destaca el desarrollo de Dua y Tuteja (2016), los cuales analizan el impacto de la crisis financiera global en los mercados dela Eurozona, China, Japón,India y Estados Unidos, los resultados sugieren incremento en la volatilidad en los mercados 
financieros de la India y Estados Unidos durante la crisis. Por otro lado, Ping, Ahmad e Ismail (2016) analizan los cambios en la volatilidad a través del tiempo entre el índice accionario estadounidense y los lingotes de oro de Malasya, también llamados KijangEmas (KE); los resultados sugieren la presencia de choques positivos y negativos, así como de efecto derrame en la volatilidad durante la crisis.

Otros autoresque utilizan la aproximación GARCH para analizar el impacto de la crisis son Ismail, Abdullah y Karim (2013) los cuales analizan el impacto de la crisis en los rendimientos de los precios de los metales preciosos; Banulescu, Hansen, Huang y Matei (2014) quienesexaminan los efectos de la crisis en los Fondos Cotizados (ETF por sus siglas en inglés) que emulan el índice S\&P 500 y; Lim y Sek (2013) analizan el impacto del tipo de cambio y precio del petróleo en la volatilidad del índice bursátil de Malasia, en periodos de inestabilidad y calma.

Cabe señalar que la mayoría de los estudios que han analizado el impacto de la crisis financiera global, incluyen en su análisis, principalmente, países desarrollados, algunos menos analizan países en desarrollo y muy pocos de ellos integran su muestra con países desarrollados y en desarrollo de diversas regiones. Es por ello, que el presente trabajo utiliza una muestra balanceada, incorporando el análisis de países emergentes y desarrollados, pertenecientes a dos continentes: americano y europeo.

En este sentido, el estudio contribuye comprobando que la crisis financiera global impactó, de manera diferenciada, el comportamiento de los mercados de valores en América y Europa, tanto emergentes como desarrollados, incrementando su volatilidad y la asimetría en la misma. Lo anterior permite evidenciar el grado de susceptibilidad de los mercados de valores ante shocks financieros locales e internacionales, en comparación con otras bolsas.

Dadas las ventajas que ofrece la modelación GARCH, el presente artículo incorpora dicha técnica para analizar las características que presenta la volatilidad accionaria de los mercados europeos y americanos, en lo referente a si la volatilidad es un proceso 
estacionario, persistente, de memoria larga y asimétrico; asimismo se prueba si es que, para dichos mercados, la crisis financiera global incrementó su volatilidad y asimetría en la misma.

\section{Metodología y series seleccionadas}

\subsection{Series seleccionadas}

Las variables empleadas son los índices bursátiles en dólares, precios de cierre diarios, de los principales mercados europeos y americanos: Irlanda (ISEQ), Francia (CAC 40), Alemania (DAX), Portugal (PSI 20), Suiza (SMI), Reino Unido (FTSE 100), Grecia (Athex 20), España (IBEX), Suecia (OMX Stockholm 30), Noruega (OSEAX) e Italia (FTSE MIB), Argentina (Mer Val), México (IPC), Canadá (S\&P TSX Composite), Brasil (BOVESPA), EUA (S\&P's 500 Index), Chile (IPSA), Colombia (IGBC-COLCAP) y Perú (IGBVL), durante el periodo primero de enero 2003 al 27 de febrero del año 2015, al ser homologadas las seriesse obtienen un total de 2455 observaciones para cada serie.

Es importante mencionar que el periodo de estudio ha sido dividido en dos subperiodos, aquel previo a la crisis y en el cual se presentaron, tanto los efectos inmediatos como los secundarios y remotos de la misma. La fecha de corte fue elegida tomando en consideración los estudios previos realizados por Acharya y Richardson (2009), Cecchetti (2008) y Taylor (2009), en los cuales se contempla que la crisis subprime y, por ende, la crisis financiera global inició el 9 de agosto del año 2007, cuando el banco francés BNP Paribas reveló importantes pérdidas no anticipadas sobre las hipotecas subprime(Greenspan, 2008) y suspendió la negociación de tres fondos por incapacidad en la valoración y liquidación de los mismos (Wu y Olson, 2015).

Con base en lo anterior, el periodo pre-crisis es aquel que contempla del $1^{\circ}$ de enero 2003 al 08 de agosto del 2007; mientras que el periodo de crisis comprende del 09 de agosto del 2007 al 27 de febrero del $2015^{2}$.

\footnotetext{
${ }^{2}$ Respecto al periodo de duración de la crisis, véase la aclaración en la nota 2
} 


\subsection{Metodología}

\subsubsection{GARCH simétrico}

Se analiza la volatilidad y el impacto que tuvieron en la misma los efectos generados por la crisis financiera global, por medio del modelo GARCH $(1,1)$ con dummy en la ecuación de la varianza. La incorporación de la variable dummy, permite probar si la volatilidad se incrementó durante el periodo de la crisis.

Para la modelación de los índices bursátiles en cuestión, las series son transformadas en rendimientos logarítmicos:

$$
R_{t}=\log \left(\frac{P_{i+1}}{P_{i}}\right), i=1,2,3 \ldots N
$$

Una de las condiciones necesarias para la aplicación del modelo es verificar que las series no presenten raíz unitaria, es decir que sean estacionarias. Para probar que dicha condición se cumple, la prueba DickeyFuller Aumentada (ADF por sus siglas en inglés) es realizada y consiste en una regresión de la primera diferencia de las series contra las series con $k$ rezagos:

$$
\Delta R_{t}=\alpha+\delta R_{t-1}+\sum_{i=1}^{p} \beta_{i} \Delta R_{t-i}+\varepsilon_{t}
$$

Donde $R_{t}$ es el rendimiento diario de las series, la hipótesis nula es $H_{0}: \delta=0$ y $H_{1}: \delta<1$. El rechazo de la hipótesis nula significa que la serie es estacionaria. Si la serie fuera no estacionaria, podría ser transformada a través de la diferenciación de la misma, para lo cual se utilizan los modelos ARMA, ARIMA y ARFIMA (López, 2004).

En cuanto a la estimación de la varianza $\sigma^{2}$ en el modelo GARCH (Bollerslev, 1986 y Taylor, 1986), la varianza condicional no sólo está en función de los cuadrados de las perturbaciones, sino que también depende de las varianzas condicionales de periodos previos. Así la varianza condicional del modelo GARCH (p, q) se especifica de la siguiente forma:

$$
h_{t}=\alpha_{0}+\sum_{i=1}^{p} \alpha_{i} \varepsilon_{t-j}^{2}+\sum_{j=1}^{q} \beta_{j} h_{t-i}, \quad p \geq 1, q \geq 1
$$


Donde es necesario que se cumplan las siguientes condiciones, para asegurar que la varianza condicional es positiva: $\alpha_{0}>0, \alpha_{1}, \alpha_{2}, \ldots \alpha_{q} \geq 0$ y $\beta_{1}, \beta_{2}, \beta_{3}, \ldots \beta_{q} \geq 0$ y se requiere que $\sum_{j=1}^{p} \alpha_{i}+\sum_{i=1}^{q} \beta_{j}<1$ y $p \geq 1, q \geq 1$ para que la varianza condicional sea convergente.

Este modelo es también denominado como GARCH simétrico, ya que asume que cambios negativos tienen el mismo impacto en magnitud que los positivos. Los coeficientes $\beta_{j}$ indican que los cambios en la varianza condicional toman largo tiempo en desaparecer, es decir que hay persistencia en la volatilidad. Cuando el coeficiente de error $\alpha_{i}$ presenta valores altos, significa que la volatilidad tiene una gran sensibilidad ante movimientos en el mercado. Si el valor de $(\alpha+\beta)$ es cercano y menor a la unidad, significa que un choque recibido en el tiempo t persistirá en periodos futuros; un alto valor del mismo, implicará que la serie tiene memoria larga (Joshi, 2012).

Para probar que los desequilibrios bursátiles originados a partir de la crisis financiera global impactaron la dinámica de los mercados de valores incrementando su volatilidad. Se introduce una variable dummy en la ecuación de la varianza:

$$
h_{t}=\alpha_{0}+\sum_{i=1}^{p} \alpha_{i} \varepsilon_{t-j}^{2}+\sum_{j=1}^{q} \beta_{j} h_{t-i}+\delta_{k} D
$$

La variable dummy (D) es un vector que toma valores de 0 en el periodo previo a la crisis $(01 / 01 / 2003$ al 08/08/2007) y valores de 1 en el periodo de crisis (09/08/2007 al 27/02/2015). Si el coeficiente de dicha variable dummy es positivo y estadísticamente significativo, indica que la crisis tuvo efectos en las series incrementando la volatilidad. Para probar el modelo se realiza la prueba ARCH-LM, si el coeficiente de la misma no es significativo la estimación es adecuada y provee buen ajuste, ya que no queda efecto ARCH en la serie, después de la estimación del modelo.

\subsubsection{GARCH asimétrico-TARCH}

Una vez que se modela la volatilidad y los cambios en la misma a partir de un modelo simétrico, se complementa la estimación por medio de un modelo GARCH asimétrico denominado TARCH (Auto regresivo con Heteroscedasticidad Condicional Umbral, por sus siglas en inglés). La principal característica y bondad de dicho modelo es que captura la 
asimetría en la volatilidad, es decir que estima de forma diferenciada los efectos provocados por los shocks positivos y negativos en la volatilidad de la serie. La ecuación de la varianza condicional para el modelo TARCH se encuentra definida de la siguiente manera:

$$
\sigma^{2}=\alpha+\sum_{i=1}^{p} \beta_{i}{\sigma_{t-j}}^{2}+\sum_{j=1}^{q} \alpha_{j} \varepsilon_{t-i}^{2}+\sum_{h=1}^{r} \gamma_{h} \varepsilon_{t-h}^{2} d_{t-h}
$$

Donde $d_{t}=1$ si $\varepsilon_{t}<0$

En este modelo si $\varepsilon_{t-i}>0$ los valores positivos del residuo se interpretan como buenas noticias, si $\varepsilon_{t-i}<0$ entonces los valores negativos del residuo representan malas noticias. El efecto sobre la varianza condicional genera que el impacto de las buenas noticias sea de $\alpha_{1}$ y las malas noticias tengan un efecto de $\alpha_{1}+\gamma_{1}$. Si $\gamma_{1}>0$, las malas noticias incrementan la volatilidad en mayor medida que las noticias positivas de la misma magnitud, lo cual se conoce como efecto apalancamiento o leverage.Si $\gamma_{1} \neq 0$, el impacto de las noticias es asimétrico (Joshi, 2012).

De igual forma que en la estimación GARCH simétrico, en la ecuación de la varianza condicional del modelo TARCH, también se introduce una variable dummy, pero en este caso para analizar el impacto de los efectos bursátiles de la crisis en la asimetría de la volatilidad. Al igual que en el modelo $\operatorname{GARCH}(1,1)$, la variable dummy (Du) es un vector que toma valores de 0 en el periodo pre-crisis y de 1 en el periodo de crisis. Si los resultados asociados a dicha variable son positivos y estadísticamente significativos, indica que la crisis incrementó la asimetría en la volatilidad, ya de por sí preexistente. La ecuación de la varianza del modelo TARCH es la siguiente:

$$
\sigma^{2}=\alpha+\sum_{j=1}^{q} \beta_{j} \sigma_{t-j}^{2}+\sum_{i=1}^{p} \alpha_{i} \varepsilon_{t-i}^{2}+\sum_{h=1}^{r} \gamma_{h} \varepsilon_{t-h}{ }^{2} d_{t-h}+\delta_{k} D u
$$

\section{Resultados}

\subsection{Análisis preliminar de las series}

En el gráfico 1 se muestran las series tanto en niveles (en color negro asociadas al eje vertical secundario) como en rendimientos (en color gris y medidos a través del eje vertical primario). Lo que se puede observar, a través de los rendimientos bursátiles, es la presencia de conjuntos de volatilidad e incrementos en la misma a partir del año 2007 y, sobre todo, en septiembre del 2008 cuando la crisis alcanzó dimensiones globales con la caída del banco LehmanBrothers. 
Nueva Época REMEF (TheMexicanJournal of Economics and Finance)

Gráfico 1. Índices bursátiles europeos en niveles y diferencias logarítmicas (US dólares)

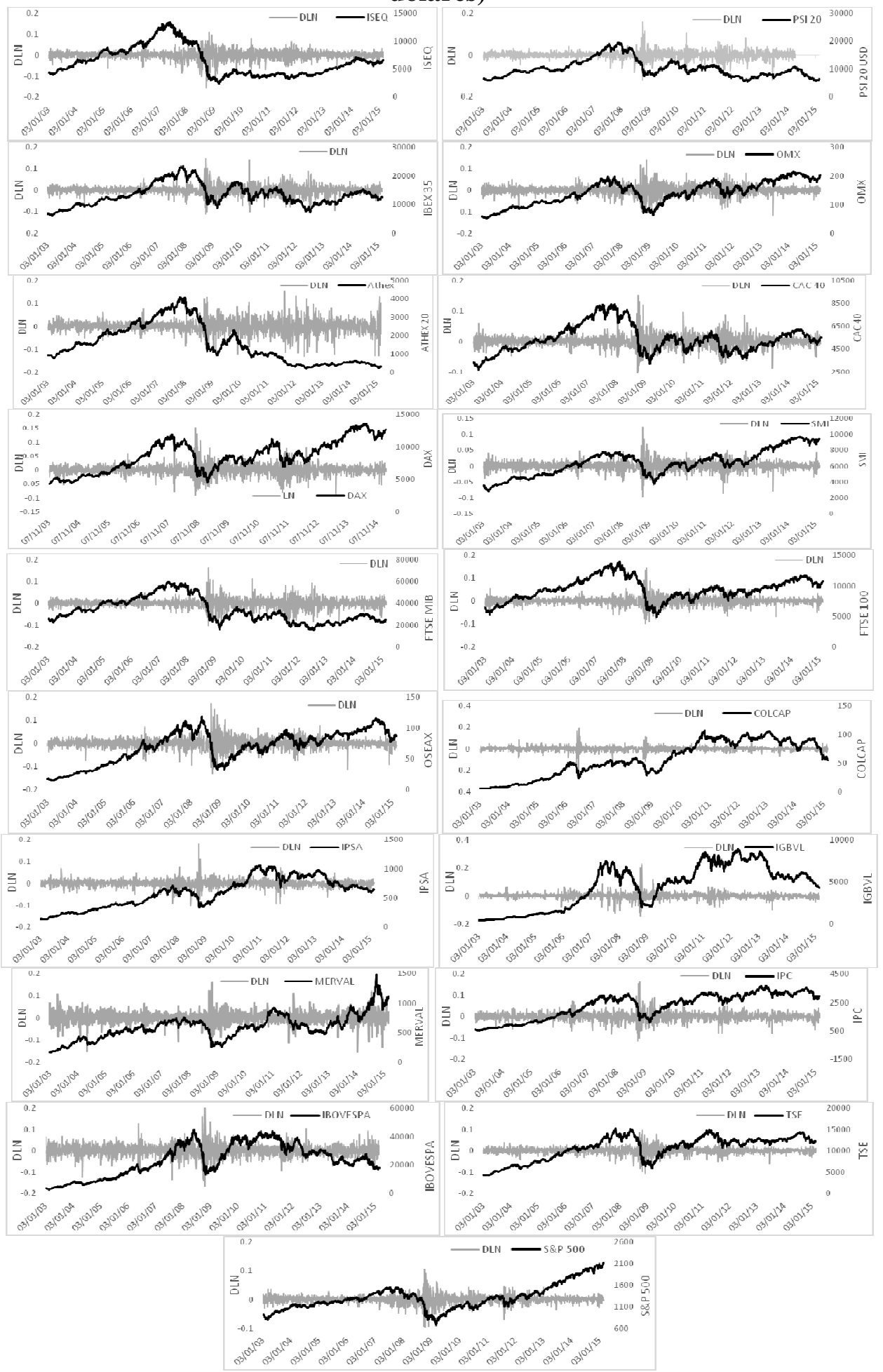


Fuente: Elaboración propia con datos de Yahoo Finanzas, Economática y Bloomberg En cuanto a las series en niveles,se observa que todos los índices bursátiles latinoamericanos presentaron una tendencia positiva hasta finales del tercer trimestre del 2008 y una tendencia negativa desde ese momento y hasta el primer trimestre del 2009, lo cual se podría deber a los efectos de la crisis en los mercados europeos y a los constantes ataques bursátiles sucedidos desde octubre del 2008, fenómeno conocido como "crash de octubre 2008". Dicho fenómeno financiero, pero sobre todo bursátil, se caracterizó por importantes variaciones en las bolsas de valores a nivel internacional ante recortes en las tasas de interés de referencia de las principales economías a nivel mundial; así como, por cambios en las calificaciones otorgadas de las agencias sobre los activos europeos, lo cual generó descontento y pánico entre los inversionistas de cartera.

En cuanto a las bolsas europeas, todas ellas presentaron caídas desde el cuarto trimestre del año 2007 y hasta el primer trimestre del 2009 (la mayoría en marzo de 2009). Las intensas variaciones en los mercados internacionales cesaron a partir de la estabilización de la economía estadounidense, por la constante inyección de liquidez, y de la aceptación de diversos planes de rescate y programas de ajuste en la Unión Europea.

La tabla 1muestra la magnitud y fecha de ocurrencia de las variaciones máximas negativas y positivas; así como, de los niveles máximos y mínimos presentados por las series. En cuanto a las variaciones máximas negativas y positivas, la mayoría de los mercados americanos presentó dichos valores durante el "crash de octubre 2008", lo cual podría ser un indicio de la transmisión de los desequilibrios de la crisis en los momentos más turbulentos de la misma.

En cuanto a los niveles máximos, las fechas en las que se presentaron no coinciden entre los mercados, lo cual se podría deber a factores y circunstancias propios de cada uno de ellos. En relación a los niveles mínimos, trece de los diecinueve mercados experimentaron a finales de enero y principios de febrero del 2003, lo cual podría deberse a la tensión que existía a nivel internacional ante el conflicto bélico en Irak. Cabe destacar, que los mercados de Portugal, Grecia e Italia presentaron niveles mínimos en los meses de junio y julio del año 2012, aparentemente, lo cual podría explicarse a partir de la expectativa de los 
mercados por la compra de deuda italiana por parte del BCE, el triunfo del partido conservador en Grecia y la aprobación de plan económico en la UE.

Tabla 1.Variaciones máximas positivas y negativas y niveles máximos y mínimos de las series

\begin{tabular}{|c|c|c|c|c|c|}
\hline \multicolumn{2}{|c|}{ Índice } & \multicolumn{2}{|c|}{ Nivel } & \multicolumn{2}{|c|}{ Variación máxima } \\
\hline & & Máximo & Mínimo & Positiva & Negativa \\
\hline \multirow{2}{*}{ PSI 20} & Cantidad & 19419.7 & 5377.0 & 0.2 & -0.1 \\
\hline & Fecha & $14 / 11 / 2007$ & $25 / 07 / 2012$ & $14 / 10 / 2008$ & $24 / 06 / 2013$ \\
\hline \multirow{2}{*}{ ISEQ } & Cantidad & 13436.7 & 2422.3 & 0.1 & -0.2 \\
\hline & Fecha & 04/06/2007 & 09/03/2009 & $04 / 11 / 2008$ & $29 / 09 / 2008$ \\
\hline \multirow{2}{*}{ CAC 40} & Cantidad & 8459.1 & 2649.1 & 0.2 & -0.1 \\
\hline & Fecha & $31 / 10 / 2007$ & $12 / 03 / 2003$ & $14 / 10 / 2008$ & $06 / 10 / 2008$ \\
\hline \multirow{2}{*}{ DAX } & Cantidad & 13643.0 & 2428.5 & 0.2 & -0.1 \\
\hline & Fecha & $03 / 07 / 2014$ & $12 / 03 / 2003$ & $14 / 10 / 2008$ & $06 / 10 / 2008$ \\
\hline \multirow{2}{*}{ SMI } & Cantidad & 9730.6 & 2759.5 & 0.1 & -0.1 \\
\hline & Fecha & $10 / 06 / 2014$ & $12 / 03 / 2003$ & $14 / 10 / 2008$ & $09 / 10 / 2008$ \\
\hline \multirow{2}{*}{ ATHEX 20} & Cantidad & 4109.8 & 211.3 & 0.2 & -0.1 \\
\hline & Fecha & $31 / 10 / 2007$ & $05 / 06 / 2012$ & $29 / 08 / 2011$ & $08 / 05 / 2012$ \\
\hline \multirow{2}{*}{ FTSE MIB } & Cantidad & 59931.3 & 14900.5 & 0.2 & -0.1 \\
\hline & Fecha & $18 / 05 / 2007$ & $24 / 07 / 2012$ & $14 / 10 / 2008$ & $06 / 10 / 2008$ \\
\hline \multirow{2}{*}{ IBEX 35} & Cantidad & 23357.4 & 6010.7 & 0.1 & -0.1 \\
\hline & Fecha & $11 / 12 / 2007$ & $12 / 03 / 2003$ & $14 / 10 / 2008$ & $10 / 10 / 2008$ \\
\hline \multirow{2}{*}{ FTSE 100} & Cantidad & 13974.2 & 4894.9 & 0.1 & -0.1 \\
\hline & Fecha & $31 / 10 / 2007$ & 09/03/2009 & $29 / 10 / 2008$ & $10 / 10 / 2008$ \\
\hline \multirow{2}{*}{ OMX } & Cantidad & 215.1 & 51.3 & 0.1 & -0.1 \\
\hline & Fecha & $06 / 03 / 2014$ & $12 / 03 / 2003$ & $09 / 12 / 2008$ & $24 / 06 / 2013$ \\
\hline \multirow{2}{*}{ OSEAX } & Cantidad & 119.0 & 14.8 & 0.2 & -0.1 \\
\hline & Fecha & $23 / 05 / 2008$ & $18 / 03 / 2003$ & $22 / 09 / 2008$ & $06 / 10 / 2008$ \\
\hline \multirow{2}{*}{ MERVAL } & Cantidad & 1487.1 & 159.5 & 0.2 & -0.2 \\
\hline & Fecha & $30 / 09 / 2014$ & $03 / 01 / 2003$ & $04 / 11 / 2008$ & $23 / 01 / 2014$ \\
\hline \multirow{2}{*}{ IPC } & Cantidad & 3680.1 & 525.1 & 0.2 & -0.1 \\
\hline & Fecha & $11 / 04 / 2013$ & $12 / 02 / 2003$ & $29 / 10 / 2008$ & $06 / 10 / 2008$ \\
\hline \multirow{2}{*}{ IBOVESPA } & Cantidad & 44673.7 & 2750.1 & 0.3 & -0.2 \\
\hline & Fecha & $30 / 05 / 2008$ & $14 / 02 / 2003$ & $14 / 10 / 2008$ & $15 / 10 / 2008$ \\
\hline \multirow{2}{*}{ TSE } & Cantidad & 15323.8 & 4220.1 & 0.1 & -0.1 \\
\hline & Fecha & $31 / 10 / 2007$ & $12 / 02 / 2003$ & $29 / 10 / 2008$ & $01 / 12 / 2008$ \\
\hline \multirow{2}{*}{ S\&P 500} & Cantidad & 2115.5 & 676.5 & 0.1 & -0.1 \\
\hline & Fecha & $24 / 02 / 2015$ & 09/03/2009 & $14 / 10 / 2008$ & $15 / 10 / 2008$ \\
\hline \multirow{2}{*}{ COLCAP } & Cantidad & 106.83 & 5.73 & 0.20 & -0.25 \\
\hline & Fecha & $04 / 11 / 2010$ & $10 / 02 / 2003$ & $16 / 06 / 2006$ & $13 / 06 / 2006$ \\
\hline \multirow{2}{*}{ IPSA } & Cantidad & 1066.7 & 132.4 & 0.2 & -0.1 \\
\hline & Fecha & $03 / 01 / 2011$ & $24 / 02 / 2003$ & $14 / 10 / 2008$ & $07 / 07 / 2008$ \\
\hline \multirow{2}{*}{ IGVBL } & Cantidad & 9007.2 & 404.8 & 0.2 & -0.1 \\
\hline & Fecha & $03 / 04 / 2012$ & $03 / 01 / 2003$ & $04 / 11 / 2008$ & $10 / 10 / 2008$ \\
\hline
\end{tabular}

Fuente: Elaboración propia con datos de Yahoo Finanzas, Economática y Bloomberg Nivel máximo y mínimo: se refiere a los niveles máximos que alcanzaron los índices bursátiles y la fecha en que sucedieron. Variación máxima y mínima: son los mayores cambios al alza y a la baja en las series 
Es importante mencionar que la bolsa de valores de Colombia es un caso particular, entre los mercados americanos, ya que presentó variaciones máximas, tanto negativas como positivas, en el periodo previo a la crisis lo cual, aparentemente, se debió a una devaluación del peso colombiano frente al dólar estadounidense de $14 \%$, impactando el comportamiento del mercado de capitales.

\subsection{Análisis de Correlación}

Como un primer acercamiento, se analiza el cambio en la correlación entre los diversos mercados de capitales bajo análisis, entre el periodo previo y durante a la crisis; asimismo, se considera al periodo 2007-2012 como aquel en el que se presentaron los efectos inmediatos de dicho fenómeno. En el tabla2 se puede apreciar que todas las bolsas incrementaron su nivel de variación conjunta con respecto al resto de los países de la muestra. Destaca el caso de Colombia y Perú, cuya correlación incrementó en $121 \%$ y 86\%, respectivamente. Igualmente, mercados como el argentino y estadounidense, cuya correlación se elevó en más de 50\%.

En cuanto a los niveles de correlación, durante el periodo que se presentaron los efectos inmediatos de la crisis (2007-2012), la mayor parte de los mercados tuvieron niveles cercanos al 0.7 , nivel reconocido en la literatura como alto. El análisis anterior, podría revelar indicios de contagio financiero entre los mercados, especialmente, en el periodo 2007-2012, fenómeno que se define comoaumento significativo del nivel de correlación entre varios mercados como consecuencia de un hecho relevante en otro u otros mercados.

Es importante mencionar que los mercados accionarios se vieron afectados durante el periodo agosto 2007- febrero 2015 por diversas circunstancias, dentro de las que destacan: los programas monetarios impulsados de octubre 2008 a octubre 2012 TARP, ZIRP y QE ${ }^{3}$, la disminución del influjo de capitales y salida de los mismos, por mayor aversión al riesgo ante la incertidumbre internacional, y menor liquidez, por la reducción de los préstamos y

\footnotetext{
${ }^{3}$ TARP (TroubleAssetRecoveryProgram), programa impulsado por el gobierno estadounidense y consistió en la inyección de recursos a bancos y grandes compañías, para la compra de activos tóxicos. ZIRP (Zero InterestRateProgram), tipo de política monetaria en el cual las tasas de interés se fijaron cercanas a cero, con el objetivo de reactivar la economía. QE (QuantitativeEasing), política monetaria poco convencional, en la cual el gobierno compra de bonos del tesoro y activos tóxicos, para inyectar liquidez a la economía.
} 
el endurecimiento de las condiciones crediticias en los mercados domésticos. Por otro lado, los mercados europeos se vieron particularmente afectados por la expectativa generada ante los planes de rescate en Irlanda, Portugal y Grecia y los programas de ajuste y planes de austeridad implementados en Europa.

Tabla 2 Análisis de correlación

\begin{tabular}{cccccc} 
País & $\begin{array}{c}\text { Correlación } \\
\text { pre-crisis }\end{array}$ & $\begin{array}{c}\text { Correlación } \\
\text { crisis }\end{array}$ & $\begin{array}{c}\text { Crecimiento } \\
\text { Correlación }\end{array}$ & $\begin{array}{c}\text { Corrprom } \\
(\mathbf{0 7 - 1 2})\end{array}$ & $\begin{array}{c}\text { Mdo } \\
\text { mayor } \\
\text { corrprom }\end{array}$ \\
\hline Francia & 0.5984 & 0.7249 & $21 \%$ & 0.7737 & Alemania \\
Italia & 0.5769 & 0.6873 & $19 \%$ & 0.7399 & Francia \\
Suecia & 0.5359 & 0.6737 & $26 \%$ & 0.7283 & Francia \\
Suiza & 0.5334 & 0.6391 & $20 \%$ & 0.6966 & Francia \\
México & 0.4387 & 0.6086 & $39 \%$ & 0.6639 & Brasil \\
EUA & 0.3783 & 0.5922 & $57 \%$ & 0.6434 & México \\
Canadá & 0.3607 & 0.533 & $48 \%$ & 0.5787 & EUA \\
Argentina & 0.3164 & 0.5032 & $59 \%$ & 0.6064 & Brasil \\
Perú & 0.2723 & 0.5057 & $86 \%$ & 0.587 & Brasil \\
Irlanda & 0.4198 & 0.6243 & $49 \%$ & 0.6778 & España \\
España & 0.577 & 0.6734 & $17 \%$ & 0.7184 & Francia \\
R. U. & 0.5663 & 0.6978 & $23 \%$ & 0.748 & Francia \\
Portugal & 0.4804 & 0.6323 & $32 \%$ & 0.6892 & Francia \\
Grecia & 0.3831 & 0.4693 & $23 \%$ & 0.5241 & Italia \\
Colombia & 0.2321 & 0.5124 & $121 \%$ & 0.5686 & Brasil \\
Alemania & 0.5768 & 0.7085 & $23 \%$ & 0.7594 & Francia \\
Brasil & 0.4066 & 0.5941 & $46 \%$ & 0.6787 & México \\
Chile & 0.4132 & 0.5599 & $36 \%$ & 0.6175 & Brasil \\
Noruega & 0.4529 & 0.6544 & $44 \%$ & 0.7173 & R. U. \\
\hline
\end{tabular}

Nota: Crecimiento correlación: se refiere a la tasa de cambio de la correlación del periodo crisis, en relación al periodo pre-crisis. CorrProm (07-12): es la correlación promedio durante el periodo inmediato de la crisis (entre los años 2007-2012). Mdo mayor correlación promedio: muestra al mercado con el cual se mantuvo mayor correlación promedio en todo el periodo de estudio.

La última columna delatabla 2 muestra el mercado con el cual las bolsas mantuvieron mayor nivel de correlación durante todo el periodo de análisis. Para los mercados europeos, Francia es el mercado con el cual la mayor parte de las bolsas europeas mantienen altos niveles de correlación; mientras que, los mercados latinoamericanos se encuentran mayormente correlacionados con el mercado brasileño. 
Lo anterior, se podría deber al tamaño de los mercados, a la influencia política y económica que Francia ejerce en la región y a los procesos de integración bursátil en los cuales enlos mercados bursátiles de Francia y Brasil se encuentran inmersos, el primero de ellos al pertenecer al mercado de valores más grande del mundo NYSE-Euronext y el segundo por las alianzas que sostiene con los mercados CME Group, Nasdaq OMX GroupInc y TSX Venture.

Igualmente, resalta el hecho de que el mercado estadounidense se correlaciona, principalmente, con el mercado mexicano, lo cual se podría deber al importante vínculo económico y comercial que guardan ambos países, influenciando el comportamiento de sus bolsas. Por otro lado, algunos factores que podrían explicar el co-moviento entre el mercado estadounidense y el resto de los países de la muestra son: la existencia de fuertes lazos financieros y de la debilidad de los sectores corporativo y bancario en países emergentes (Didier, Love y Martinez, 2011).

Del análisis de correlación se obtienen indicios de cambios en la dinámica de los índices bursátiles, sugiriendo la transmisión de los efectos de la crisis financiera global entre los mercados. Con el objetivo de saber si estos efectos impactaron el comportamiento de la volatilidad, incrementándola y haciendo mayor su asimetría, en el largo plazo se aplican los modelos GARCH y TARCH.

\subsection{Modelo GARCH con dummy en la ecuación de varianza}

En las tablas 3 y 4 son presentados los estadísticos básicos de las series en el periodo precrisis y el periodo de la crisis, respectivamente. Del análisis preliminar de los datos, es posible concluir que la volatilidad, medida como la desviación estándar, es mayor en el periodo de crisis, excepto para el caso de Colombia, ya que en junio del 2006 se presentó una gran devaluación del peso colombiano frente al dólar estadounidense, lo que afectó de manera importante el mercado bursátil.

En el periodo pre-crisis la distribución de los rendimientos de todas las series es sesgada de manera negativa, excepto para algunos mercados como el francés, alemán, inglés, italiano, 
suizo, chileno, mexicano y peruano, en los cuales su distribución tomó un sesgo positivo. Los valores referentes a la curtósis, sugieren que la distribución de las series en el periodo pre crisis y crisis, es de carácter leptocúrtico, es decir, presentan alta concentración en los valores centrales (apuntamiento) y registran colas pesadas.

Tabla 3. Estadísticos básicos periodo pre-crisis 01/01/2003- 08/08/2007

\begin{tabular}{ccccccccc} 
País & D. E. & Media & Curtósis & Sesgo & JarqueBera & \multicolumn{2}{c}{$\begin{array}{c}\text { ARCH } \\
\text { Estadístico F }\end{array}$} & Probabilidad \\
\hline Grecia & 0.0142 & 0.0014 & 5.4167 & -0.0567 & $229.0158^{*}$ & $28.40(1)^{*}$ & 0.00 \\
Francia & 0.0118 & 0.0009 & 5.0975 & -0.1156 & $174.2303^{*}$ & $34.62(1)^{*}$ & 0.00 \\
Alemania & 0.0132 & 0.0012 & 4.9793 & -0.0897 & $154.5413^{*}$ & $7.52(1)^{*}$ & 0.01 \\
R. U. & 0.0101 & 0.0007 & 5.2758 & -0.1900 & $208.2956^{*}$ & $45.62(1)^{*}$ & 0.00 \\
Italia & 0.0103 & 0.0008 & 4.3290 & -0.4139 & $95.91271^{*}$ & $12.00(1)^{*}$ & 0.00 \\
España & 0.0231 & 0.0023 & 5.4599 & -0.3863 & $260.1003^{*}$ & $3.55(1)^{* * *}$ & 0.06 \\
Brasil & 0.0109 & 0.0012 & 5.1586 & -0.4239 & $210.4243^{*}$ & $35.06(1)^{*}$ & 0.00 \\
Irlanda & 0.0114 & 0.0011 & 6.0452 & -0.6750 & $434.1319^{*}$ & $13.94(2)^{*}$ & 0.00 \\
Argentina & 0.0209 & 0.0015 & 7.8867 & -0.5650 & $984.2736^{*}$ & $7.68(1)^{*}$ & 0.01 \\
Suecia & 0.0135 & 0.0012 & 5.4716 & -0.2943 & $252.5568^{*}$ & $27.07(1)^{*}$ & 0.00 \\
Noruega & 0.0146 & 0.0018 & 6.1747 & -0.7567 & $483.9505^{*}$ & $9.70(1)^{*}$ & 0.00 \\
Portugal & 0.0092 & 0.0011 & 4.6067 & -0.2456 & $110.4349^{*}$ & $3.14(1)^{* * *}$ & 0.08 \\
Suiza & 0.0105 & 0.0008 & 4.7434 & -0.2879 & $131.888^{*}$ & $3.23(1)^{* * *}$ & 0.07 \\
EUA & 0.0084 & 0.0005 & 4.3735 & -0.0342 & $73.99635^{*}$ & $5.81(2)^{*}$ & 0.00 \\
Canadá & 0.0098 & 0.0012 & 4.2862 & -0.3532 & $84.2478^{*}$ & $3.48(3)^{* *}$ & 0.02 \\
Colombia & 0.0229 & 0.0023 & 30.1590 & -1.0243 & $29023.19^{*}$ & $18.04(1)^{*}$ & 0.00 \\
Chile & 0.0142 & 0.0031 & 8.9560 & -0.0321 & $1388.085^{*}$ & $3.507479(1)^{*}$ & 0.00 \\
México & 0.0145 & 0.0016 & 5.9237 & -0.2688 & $345.745^{*}$ & $9.78(1)^{*}$ & 0.00 \\
Perú & 0.0121 & 0.0016 & 6.1493 & -0.4834 & $424.6189^{*}$ & $237.76(1)^{* * *}$ & 0.06 \\
\hline
\end{tabular}

Los valores reportados son estadísticamente significativos *** y *** al 1, 5 y 10\%. Los números entre paréntesis indican el número de rezagos utilizados para la prueba $\mathrm{ARCH}$.

Nota: La prueba estadística ARCH LM es el multiplicador de Lagrange usado para la detección del efecto ARCH. Bajo la hipótesis nula de no heteroscedasticidad, dicho termino se distribuye como $\lambda^{2}(k)$.

En cuanto al rendimiento medio de las series, sufrió una importante disminución en el periodo de crisis, en referencia al periodo previo, llegando en algunos casos a ser incluso negativo, lo cual significa que hubo pérdidas para los inversionistas que mantuvieron posición en dichos mercados (Chile, Canadá, Portugal, Noruega, Irlanda, Brasil, España, Italia, Reino Unido, Grecia y Francia). En cuanto a la probabilidad reportada del estadístico JarqueBera en las tablas 3 y 4, permite el rechazo de la hipótesis de distribución normal al $1 \%$ de significancia 
Tabla 4. Estadísticos básicos periodo de crisis 09/08/2007- 27/02/2015

\begin{tabular}{|c|c|c|c|c|c|c|c|}
\hline \multirow{2}{*}{ País } & \multirow{2}{*}{ D. E. } & \multirow{2}{*}{ Media } & \multirow{2}{*}{ Curtósis } & \multirow{2}{*}{ Sesgo } & \multirow{2}{*}{ JarqueBera } & \multicolumn{2}{|c|}{ ARCH (1) } \\
\hline & & & & & & Estadístico F & Probabilidad \\
\hline Grecia & 0.032 & -0.0016470 & 5.512 & -0.046 & $399.04 *$ & $13.18^{*}$ & 0.00 \\
\hline Francia & 0.021 & -0.0002110 & 8.491 & 0.139 & $1909.35^{*}$ & $94.96^{*}$ & 0.00 \\
\hline Alemania & 0.021 & 0.0001440 & 8.679 & 0.145 & $2042.45^{*}$ & $81.47 *$ & 0.00 \\
\hline R. U. & 0.018 & -0.0001040 & 11.425 & 0.023 & $4484.12 *$ & $132.66^{*}$ & 0.00 \\
\hline Italia & 0.024 & -0.0005070 & 7.072 & 0.023 & $1047.45^{*}$ & $53.01 *$ & 0.00 \\
\hline España & 0.023 & -0.0003060 & 7.473 & -0.009 & $1263.99 *$ & $81.06^{*}$ & 0.00 \\
\hline Brasil & 0.029 & -0.0002870 & 13.501 & 0.395 & $7004.79 *$ & $129.01 *$ & 0.00 \\
\hline Irlanda & 0.022 & -0.0003680 & 8.425 & -0.802 & $2021.37 *$ & $29.60^{*}$ & 0.00 \\
\hline Argentina & 0.025 & 0.0003260 & 8.909 & -0.462 & $2259.64 *$ & $64.78^{*}$ & 0.00 \\
\hline Suecia & 0.023 & 0.0000801 & 6.872 & -0.022 & $947.13^{*}$ & $67.72 *$ & 0.00 \\
\hline Noruega & 0.024 & -0.0000530 & 8.942 & -0.137 & $2234.92 *$ & $128.02 *$ & 0.00 \\
\hline Portugal & 0.020 & -0.0006900 & 9.999 & -0.058 & $3095.24 *$ & $13.37 *$ & 0.00 \\
\hline Suiza & 0.015 & 0.0001730 & 10.201 & 0.010 & $3275.58 *$ & $56.43^{*}$ & 0.00 \\
\hline EUA & 0.015 & 0.0002380 & 10.371 & -0.454 & $3483.70 *$ & $172.03 *$ & 0.00 \\
\hline Canadá & 0.016 & -0.0000319 & 9.703 & -0.484 & $2897.44 *$ & $191.95^{*}$ & 0.00 \\
\hline Colombia & 0.017 & 0.0000157 & 10.851 & -0.558 & $3971.67 *$ & $223.31 *$ & 0.00 \\
\hline Chile & 0.022 & -0.0003300 & 20.680 & 0.425 & $19791.25^{*}$ & $52.16^{*}$ & 0.00 \\
\hline México & 0.020 & 0.0000574 & 11.086 & 0.256 & $4146.74 *$ & $103.35^{*}$ & 0.00 \\
\hline Perú & 0.018 & 0.0000245 & 16.369 & 0.100 & $11292.55^{*}$ & $95.67 *$ & 0.00 \\
\hline
\end{tabular}

Los resultados de la prueba ARCH LM indican la presencia de efecto ARCH, para todas y cada una de las series bajo estudio, ya que la probabilidad asociada a los mismos es menor a 0.1 , en el periodo previo a la crisis y menor a 0.01 en el periodo de crisis. Dado que el modelo GARCH es apto para la modelación de series leptocúrticas, heteroscedásticas y asimétricas. Este modelo permite describir y analizar el comportamiento de los datos presentados.

\subsection{Resultados del modelo GARCH con dummy en la varianza}

Una vez que se analizaron los estadísticos básicos de las series y se probó la pertinencia de los modelos GARCH para su análisis, se realiza la prueba de raíces unitarias para todas las series en el periodo pre crisis y crisis. Los resultados de la prueba DickeyFuller Aumentada se reportan en la tabla 5 y sugieren el rechazo de la hipótesis nula de presencia de raíz 
unitaria, es decir se comprueba la estacionaridad de las series financieras bajo estudio, condición necesaria para la estimación del modelo.

Tabla 5. Pruebas de raíces unitarias para los rendimientos diarios bursátiles

\begin{tabular}{ccccc}
\hline & \multicolumn{2}{c}{ Antes de la crisis } & \multicolumn{2}{c}{ Durante y después crisis } \\
& Niveles & Primeras Diferencias & Niveles & Primeras Diferencias \\
\hline Grecia & $-28.96(0.00)^{*}$ & $-16.60(0.00)^{*}$ & $-38.49(0.00)^{*}$ & $-18.64(0.00)^{*}$ \\
Francia & $-18.79(0.00)^{*}$ & $-15.41(0.00)^{*}$ & $-41.21(0.00)^{*}$ & $-16.36(0.00)^{*}$ \\
Alemania & $-31.27(0.00)^{*}$ & $-15.48(0.00)^{*}$ & $-39.37(0.00)^{*}$ & $-16.34(0.00)^{*}$ \\
R. U. & $-33.31(0.00)^{*}$ & $-15.58(0.00)^{*}$ & $-41.18(0.00)^{*}$ & $-21.57(0.00)^{*}$ \\
Italia & $-19.64(0.00)^{*}$ & $-14.89(0.00)^{*}$ & $-39.43(0.00)^{*}$ & $-20.85(0.00)^{*}$ \\
España & $-18.28(0.00)^{*}$ & $-19.01(0.00)^{*}$ & $-38.57(0.00)^{*}$ & $-18.48(0.00)^{*}$ \\
Brasil & $-30.52(0.00)^{*}$ & $-16.97(0.00)^{*}$ & $-38.97(0.00)^{*}$ & $-19.82(0.00)^{*}$ \\
Irlanda & $-30.08(0.00)^{*}$ & $-14.20(0.00)^{*}$ & $-38.65(0.00)^{*}$ & $-17.77(0.00)^{*}$ \\
Argentina & $-32.24(0.00)^{*}$ & $-17.62(0.00)^{*}$ & $-37.66(0.00)^{*}$ & $-17.81(0.00)^{*}$ \\
Suecia & $-18.31(0.00)^{*}$ & $-15.73(0.00)^{*}$ & $-40.72(0.00)^{*}$ & $-16.74(0.00)^{*}$ \\
Noruega & $-29.28(0.00)^{*}$ & $-15.92(0.00)^{*}$ & $-40.39(0.00)^{*}$ & $-16.75(0.00)^{*}$ \\
Portugal & $-29.32(0.00)^{*}$ & $-19.47(0.00)^{*}$ & $-38.03(0.00)^{*}$ & $-18.87(0.00)^{*}$ \\
Suiza & $-31.41(0.00)^{*}$ & $-15.17(0.00)^{*}$ & $-42.07(0.00)^{*}$ & $-17.08(0.00)^{*}$ \\
EUA & $-32.46(0.00)^{*}$ & $-16.59(0.00)^{*}$ & $-43.26(0.00)^{*}$ & $-21.41(0.00)^{*}$ \\
Canadá & $-29.98(0.00)^{*}$ & $-14.69(0.00)^{*}$ & $-35.25(0.00)^{*}$ & $-17.16(0.00)^{*}$ \\
Colombia & $-29.90(0.00)^{*}$ & $-13.98(0.00)^{*}$ & $-13.31(0.00)^{*}$ & $-23.05(0.00)^{*}$ \\
Chile & $-25.66(0.00)^{*}$ & $-14.61(0.00)^{*}$ & $-37.08(0.00)^{*}$ & $-16.80(0.00)^{*}$ \\
México & $-29.48(0.00)^{*}$ & $-18.26(0.00)^{*}$ & $-35.19(0.00)^{*}$ & $-18.75(0.00)^{*}$ \\
Perú & $-27.96(0.00)^{*}$ & $-19.55(0.00)^{*}$ & $-35.74(0.00)^{*}$ & $-18.68(0.00)^{*}$ \\
\hline & & & &
\end{tabular}

El valor crítico de MacKinnon al nivel de significancia del 1\% es -3.44. Entre paréntesis se encuentran los valores de probabilidad asociados a los estadísticos.

Probada la condición de estacionaridad, se estima el modelo GARCH por medio del logaritmo de optimización Marquardt, utilizando como criterio para la elección de modelo óptimo el método de máxima verosimilitud. Derivado del análisis de máxima verosimilitud y con base en los resultados del modelo, fue elegido el GARCH $(1,1)$, ya que el resto de los modelos presenta coeficientes negativos $\alpha_{1}$ y $\beta_{1}$, para toda $i \neq 0$, o no significativos al menos al 90\% de confianza. Los resultados del modelo GARCH $(1,1)$ se encuentran en la tabla 6. 
Revista Mexicana de Economía y Finanzas, Vol. 12, No. 3, (2017), pp.1-27

Tabla 6. Coeficientes de los modelos GARCH periodo 01/01/2003- 27/02/2015

\begin{tabular}{|c|c|c|c|c|c|c|c|c|}
\hline Países & $\alpha 0$ & $\alpha 1$ & $\beta 1$ & $\alpha 1+\beta 1$ & Dummy & Prob & $\begin{array}{l}\text { ARCH } \\
\text { LM (1) }\end{array}$ & Prob \\
\hline Colombia & $\begin{array}{l}3.00 \mathrm{E}-05 \\
(0.000)^{*}\end{array}$ & $\begin{array}{l}0.2119 \\
(0.000)\end{array}$ & $\begin{array}{l}0.7132 \\
(0.000)\end{array}$ & 0.9252 & $-7.70 \mathrm{E}-06$ & $(0.006)$ & 0.1922 & $(0.6611)$ \\
\hline Chile & $\begin{array}{c}6.73 \mathrm{E}-06 \\
(0.000)\end{array}$ & $\begin{array}{l}0.1265 \\
(0.000)\end{array}$ & $\begin{array}{l}0.8448 \\
(0.000)\end{array}$ & 0.9713 & $1.74 \mathrm{E}-06$ & $(0.0476)$ & 0.2563 & $(0.6127)$ \\
\hline México & $\begin{array}{c}7.47 \mathrm{E}-06 \\
(0.000)\end{array}$ & $\begin{array}{l}0.0946 \\
(0.000)\end{array}$ & $\begin{array}{l}0.8782 \\
(0.000)\end{array}$ & 0.9728 & $1.24 \mathrm{E}-06$ & $(0.2198)$ & 0.0078 & $(0.9297)$ \\
\hline Perú & $\begin{array}{c}4.59 \mathrm{E}-06 \\
(0.000)\end{array}$ & $\begin{array}{l}0.1437 \\
(0.000)\end{array}$ & $\begin{array}{l}0.8472 \\
(0.000)\end{array}$ & 0.9910 & 4.43E-06 & $(0.000)$ & 0.0002 & $(0.9887)$ \\
\hline EUA & $\begin{array}{c}2.52 \mathrm{E}-06 \\
(0.000)\end{array}$ & $\begin{array}{l}0.1027 \\
(0.000)\end{array}$ & $\begin{array}{l}0.8746 \\
(0.000)\end{array}$ & 0.9773 & $7.20 \mathrm{E}-07$ & $(0.0732)$ & 3.3912 & $(0.0657)$ \\
\hline Canadá & $\begin{array}{c}1.93 \mathrm{E}-06 \\
(0.000)\end{array}$ & $\begin{array}{l}0.0668 \\
(0.000)\end{array}$ & $\begin{array}{l}0.9236 \\
(0.000)\end{array}$ & 0.9905 & $-3.17 \mathrm{E}-07$ & $(0.3007)$ & 0.1118 & $(0.7381)$ \\
\hline Grecia & $\begin{array}{c}8.34 \mathrm{E}-06 \\
(0.000)\end{array}$ & $\begin{array}{l}0.0831 \\
(0.000)\end{array}$ & $\begin{array}{l}0.8805 \\
(0.000)\end{array}$ & 0.9636 & $2.87 \mathrm{E}-05$ & $(0.000)$ & 0.0012 & $(0.9720)$ \\
\hline Francia & $\begin{array}{c}3.31 \mathrm{E}-06 \\
(0.000)\end{array}$ & $\begin{array}{l}0.0848 \\
(0.000)\end{array}$ & $\begin{array}{l}0.8968 \\
(0.000)\end{array}$ & 0.9816 & $3.69 \mathrm{E}-06$ & $(0.0002)$ & 0.3299 & $(0.5657)$ \\
\hline Alemania & $\begin{array}{c}3.81 \mathrm{E}-06 \\
(0.000)\end{array}$ & $\begin{array}{l}0.0839 \\
(0.000)\end{array}$ & $\begin{array}{l}0.8960 \\
(0.000)\end{array}$ & 0.9799 & $3.41 \mathrm{E}-06$ & $(0.0001)$ & 0.9432 & $(0.3316)$ \\
\hline R. U. & $\begin{array}{c}2.46 \mathrm{E}-06 \\
(0.000)\end{array}$ & $\begin{array}{l}0.0880 \\
(0.000)\end{array}$ & $\begin{array}{l}0.8940 \\
(0.000)\end{array}$ & 0.9820 & $1.71 \mathrm{E}-06$ & $(0.0003)$ & 0.4499 & $(0.5025)$ \\
\hline Italia & $\begin{array}{c}3.28 \mathrm{E}-06 \\
(0.000)\end{array}$ & $\begin{array}{l}0.0851 \\
(0.000)\end{array}$ & $\begin{array}{l}0.8899 \\
(0.000)\end{array}$ & 0.9751 & $8.67 \mathrm{E}-06$ & $(0.000)$ & 0.0402 & $(0.8411)$ \\
\hline España & $\begin{array}{c}3.68 \mathrm{E}-06 \\
(0.000)\end{array}$ & $\begin{array}{l}0.0811 \\
(0.000)\end{array}$ & $\begin{array}{l}0.8955 \\
(0.000)\end{array}$ & 0.9766 & $6.80 \mathrm{E}-06$ & $(0.000)$ & 0.7199 & $(0.3962)$ \\
\hline Brasil & $\begin{array}{c}1.82 \mathrm{E}-05 \\
(0.000)\end{array}$ & $\begin{array}{l}0.0858 \\
(0.000)\end{array}$ & $\begin{array}{l}0.8878 \\
(0.000)\end{array}$ & 0.9736 & $-2.44 \mathrm{E}-06$ & $(0.2961)$ & 0.5803 & $(0.4463)$ \\
\hline Irlanda & $\begin{array}{c}2.89 \mathrm{E}-06 \\
(0.000)\end{array}$ & $\begin{array}{l}0.0737 \\
(0.000)\end{array}$ & $\begin{array}{l}0.9134 \\
(0.000)\end{array}$ & 0.9872 & $1.83 \mathrm{E}-06$ & $(0.0058)$ & 0.6795 & $(0.4099)$ \\
\hline Argentina & $\begin{array}{c}2.68 \mathrm{E}-05 \\
(0.000)\end{array}$ & $\begin{array}{l}0.1034 \\
(0.000)\end{array}$ & $\begin{array}{l}0.8386 \\
(0.000)\end{array}$ & 0.9420 & 7.57E-06 & $(0.0002)$ & 0.0129 & $(0.9096)$ \\
\hline Suecia & $\begin{array}{c}3.22 \mathrm{E}-06 \\
(0.000)\end{array}$ & $\begin{array}{l}0.0643 \\
(0.000)\end{array}$ & $\begin{array}{l}0.9232 \\
(0.000)\end{array}$ & 0.9875 & $1.83 \mathrm{E}-06$ & $(0.0024)$ & 0.0550 & $(0.8146)$ \\
\hline Noruega & $\begin{array}{c}5.16 \mathrm{E}-06 \\
(0.000)\end{array}$ & $\begin{array}{l}0.0754 \\
(0.000)\end{array}$ & $\begin{array}{l}0.9085 \\
(0.000)\end{array}$ & 0.9839 & $1.95 \mathrm{E}-06$ & (0.0139) & 0.0191 & $(0.8900)$ \\
\hline Portugal & $\begin{array}{c}5.93 \mathrm{E}-06 \\
(0.000)\end{array}$ & $\begin{array}{l}0.0881 \\
(0.000)\end{array}$ & $\begin{array}{l}0.8484 \\
(0.000)\end{array}$ & 0.9365 & $1.81 \mathrm{E}-05$ & $(0.000)$ & 0.4582 & $(0.4985)$ \\
\hline Suiza & $\begin{array}{c}3.11 \mathrm{E}-06 \\
(0.000)\end{array}$ & $\begin{array}{l}0.0738 \\
(0.000)\end{array}$ & $\begin{array}{l}0.9023 \\
(0.000)\end{array}$ & 0.9761 & $1.41 \mathrm{E}-06$ & $(0.0056)$ & 0.8610 & $(0.3535)$ \\
\hline
\end{tabular}

*valores entre paréntesis representan las probabilidades

El modelo GARCH es probado a partir del estadístico ARCH-LM, el cual presenta valores de probabilidad mayores al 0.05, lo cual indica ausencia del efecto ARCH después de la modelación, comprobando que la estimación es adecuada y provee buen ajuste. Los resultados de la estimación indican que todos los parámetros son positivos y estadísticamente significativos, con una probabilidad menor a 0.05 .

Igualmente, se denota que el proceso $\mathrm{ARCH}$ es de carácter estacionario, es decir que la varianza no crece de forma indefinida, ya que en la suma $\alpha_{1}+\beta_{1}$ tiene un resultado muy cercano e inferiora la unidad. Igualmente, los coeficientes del modelo GARCH que se 
encuentran en la tabla 6 sugieren que existe persistencia en la volatilidad, es decir, la volatilidad no decae rápidamente, sino que tiende a permanecer y su efecto se desvanece poco a poco, ya que el coeficiente de retardo de la varianza condicional $\beta_{1}$ es mayor que el coeficiente de error $\alpha_{1}$.

Los coeficientes de las variables dummy introducidas en la ecuación de la varianza (Dummy) son positivasy son significativas estadísticamente al $95 \%$ de confianza, para la mayoría de los mercados, excepto para el caso de Colombia, Canadá y Brasil, que sus coeficientes son negativos, ypara los mercados de México, Canadá y Brasil, que sus coeficientes no son estadísticamente significativos. Lo anterior sugiere que en todos los mercados europeos y en Argentina, Perú, Chile y Estados Unidos la volatilidad se incrementó a partir de los efectos de la crisis financiera global, por lo cual, dichos mercados se encuentran mayormente integrados a la dinámica económica y, sobre todo, financiera internacional.

A partir de dicha información y dada la naturaleza de las series financieras, especialmente en lo referente a la asimetría en la volatilidad, se aplica un modelo TARCH para el análisis de las series financieras bajo estudio, con el propósito de probar asimetría en la volatilidad y cambios en la misma a partir de la crisis financiera.

\subsection{Modelo TARCH con dummy en la ecuación de varianza}

A lo largo de la literatura empírica se ha acopiado evidencia de asimetría en la volatilidad de las series bursátiles y financieras. Con el objetivo de probar que los impactos o noticias negativas tienen mayor efecto en la volatilidad de los índices latinoamericanos que las noticias positivas de la misma magnitud, se aplica un modelo TARCH que permita capturar dicho efecto. Dentro del modelo se introduce una variable dummy en la ecuación de la varianza condicional, para saber si la asimetría en las series ha aumentado a partir de los efectos de la crisis. Los resultados de la estimación del modelo se encuentran en la tabla 7.

En la tabla 7 se observa que el termino de apalancamiento $(\gamma)$ representado por $\operatorname{RESID}<0$ ARCH (1), es en todos los casos positivo y estadísticamente significativo, lo cual refuerza el supuesto de que los impactos negativos tienen mayor efecto sobre la volatilidad que los 
impactos positivos de la misma magnitud. Las noticias buenas tienen un impacto de $\alpha_{1}$, mientras las malas noticias tienen un impacto $\alpha_{1}+\gamma$, es decir, su impacto es mayor que el de las buenas noticias en todos los mercados financieros aquí analizados. En este sentido, los mercados con mayor asimetría en su volatilidad son el canadiense, peruano, irlandés, griego y noruego, mientras que los mercados que presentan menor asimetría son los de Chile, Argentina, Portugal, Estados Unidos y Colombia. En general, se aporta evidencia de que todos los mercados analizados reportan asimetría en su volatilidad, siendo las malas noticias o impactos negativos los que producen mayores efectos en la volatilidad, en relación a las buenas noticias.

Tabla 7. Coeficientes de los modelos TARCH periodo 01/01/2003- 27/02/2015

\begin{tabular}{|c|c|c|c|c|c|c|c|c|c|}
\hline Coeficientes & $\alpha_{0}$ & $\alpha_{1}$ & $\beta_{1}$ & $\begin{array}{c}\text { RESID }<0 \text { ARCH (1) } \\
\gamma\end{array}$ & $\alpha_{1}+\gamma$ & Du & Prob & $\begin{array}{c}\text { ARCH } \\
\text { LM test }\end{array}$ & Prob \\
\hline Colombia & $\begin{array}{c}3.69 \mathrm{E}-05 \\
(0.000)\end{array}$ & $\begin{array}{l}0.0861 \\
(0.000)\end{array}$ & $\begin{array}{l}0.2371 \\
(0.000)\end{array}$ & $\begin{array}{l}0.7005 \\
(0.000)\end{array}$ & 0.7865 & $-1.20 \mathrm{E}-05$ & $(0.000)$ & 0.0381 & $(0.8454)$ \\
\hline Chile & $\begin{array}{c}9.99 \mathrm{E}-06 \\
(0.000)\end{array}$ & $\begin{array}{l}0.0538 \\
(0.000)\end{array}$ & $\begin{array}{l}0.1181 \\
(0.000)\end{array}$ & $\begin{array}{l}0.8385 \\
(0.000)\end{array}$ & 0.8923 & $7.18 \mathrm{E}-07$ & $(0.3835)$ & 0.7413 & $(0.3893)$ \\
\hline México & $\begin{array}{c}6.70 \mathrm{E}-06 \\
(0.000)\end{array}$ & $\begin{array}{c}0.0036 \\
(0.5261)\end{array}$ & $\begin{array}{l}0.1309 \\
(0.000)\end{array}$ & $\begin{array}{l}0.9079 \\
(0.000)\end{array}$ & 0.9115 & $-1.09 \mathrm{E}-06$ & $(0.1346)$ & 0.4542 & $(0.5004)$ \\
\hline Perú & $\begin{array}{c}5.10 \mathrm{E}-06 \\
(0.000)\end{array}$ & $\begin{array}{l}0.1116 \\
(0.000)\end{array}$ & $\begin{array}{l}0.0596 \\
(0.000)\end{array}$ & $\begin{array}{l}0.8481 \\
(0.000)\end{array}$ & 0.9597 & $3.52 \mathrm{E}-06$ & $(0.0004)$ & 0.0365 & $(0.8485)$ \\
\hline EUA & $\begin{array}{c}1.17 \mathrm{E}-05 \\
(0.000)\end{array}$ & $\begin{array}{l}0.1778 \\
(0.000)\end{array}$ & $\begin{array}{l}0.1997 \\
(0.000)\end{array}$ & $\begin{array}{l}0.7033 \\
(0.000)\end{array}$ & 0.8810 & 5.01E-07 & $(0.7707)$ & 0.7476 & $(0.4548)$ \\
\hline Canadá & $\begin{array}{c}2.29 \mathrm{E}-06 \\
(0.000)\end{array}$ & $\begin{array}{l}0.0357 \\
(0.000)\end{array}$ & $\begin{array}{l}0.0528 \\
(0.000)\end{array}$ & $\begin{array}{l}0.9245 \\
(0.000)\end{array}$ & 0.9602 & $-6.24 \mathrm{E}-07$ & $(0.0381)$ & 0.2126 & $(0.6447)$ \\
\hline Grecia & $\begin{array}{c}9.05 \mathrm{E}-06 \\
(0.000)\end{array}$ & $\begin{array}{l}0.0666 \\
(0.000)\end{array}$ & $\begin{array}{c}0.0316 \\
(0.0137)\end{array}$ & $\begin{array}{l}0.8782 \\
(0.000)\end{array}$ & 0.9449 & $2.88 \mathrm{E}-05$ & $(0.000)$ & 0.0001 & (0.9917) \\
\hline Francia & $\begin{array}{c}4.47 \mathrm{E}-06 \\
(0.000)\end{array}$ & $\begin{array}{c}0.0092 \\
(0.3296)\end{array}$ & $\begin{array}{l}0.1250 \\
(0.000)\end{array}$ & $\begin{array}{l}0.9025 \\
(0.000)\end{array}$ & 0.9117 & $3.21 \mathrm{E}-06$ & $(0.000)$ & 1.1196 & $(0.2901)$ \\
\hline Alemania & $\begin{array}{c}4.76 \mathrm{E}-06 \\
(0.000)\end{array}$ & $\begin{array}{c}0.0181 \\
(0.0899)\end{array}$ & $\begin{array}{l}0.1020 \\
(0.000)\end{array}$ & $\begin{array}{l}0.9063 \\
(0.000)\end{array}$ & 0.9244 & $2.24 \mathrm{E}-06$ & $(0.0069)$ & 3.3488 & $(0.0674)$ \\
\hline R. U. & $\begin{array}{c}3.13 \mathrm{E}-06 \\
(0.000)\end{array}$ & $\begin{array}{c}0.0193 \\
(0.0285)\end{array}$ & $\begin{array}{l}0.1178 \\
(0.000)\end{array}$ & $\begin{array}{l}0.8985 \\
(0.000)\end{array}$ & 0.9178 & $1.10 \mathrm{E}-06$ & $(0.0228)$ & 1.8128 & $(0.1783)$ \\
\hline Italia & $\begin{array}{c}3.88 \mathrm{E}-06 \\
(0.000)\end{array}$ & $\begin{array}{c}0.0176 \\
(0.0657)\end{array}$ & $\begin{array}{l}0.1027 \\
(0.000)\end{array}$ & $\begin{array}{l}0.8999 \\
(0.000)\end{array}$ & 0.9175 & $7.51 \mathrm{E}-06$ & $(0.000)$ & 0.0315 & $(0.8591)$ \\
\hline España & $\begin{array}{c}4.97 \mathrm{E}-06 \\
(0.000)\end{array}$ & $\begin{array}{c}0.0129 \\
(0.1452)\end{array}$ & $\begin{array}{l}0.1108 \\
(0.000)\end{array}$ & $\begin{array}{l}0.9015 \\
(0.000)\end{array}$ & 0.9144 & $5.09 \mathrm{E}-06$ & $(0.000)$ & 1.4088 & $(0.2354)$ \\
\hline Brasil & $\begin{array}{c}2.23 \mathrm{E}-05 \\
(0.000)\end{array}$ & $\begin{array}{c}0.0098 \\
(0.2551)\end{array}$ & $\begin{array}{l}0.1299 \\
(0.000)\end{array}$ & $\begin{array}{l}0.8932 \\
(0.000)\end{array}$ & 0.9031 & $-5.71 \mathrm{E}-06$ & $(0.0094)$ & 2.7013 & $(0.0673)$ \\
\hline Irlanda & $\begin{array}{c}3.59 \mathrm{E}-06 \\
(0.000)\end{array}$ & $\begin{array}{l}0.0455 \\
(0.000)\end{array}$ & $\begin{array}{l}0.0499 \\
(0.000)\end{array}$ & $\begin{array}{l}0.9101 \\
(0.000)\end{array}$ & 0.9556 & $2.17 \mathrm{E}-06$ & $(0.000)$ & 1.1316 & $(0.2875)$ \\
\hline Argentina & $\begin{array}{c}3.44 \mathrm{E}-05 \\
(0.000)\end{array}$ & $\begin{array}{l}0.0756 \\
(0.000)\end{array}$ & $\begin{array}{l}0.0615 \\
(0.000)\end{array}$ & $\begin{array}{l}0.8165 \\
(0.000)\end{array}$ & 0.8920 & 8.89E-06 & $(0.000)$ & 0.0089 & $(0.9251)$ \\
\hline Suecia & $\begin{array}{c}4.24 \mathrm{E}-06 \\
(0.000)\end{array}$ & $\begin{array}{c}0.0049 \\
(0.5144)\end{array}$ & $\begin{array}{l}0.0984 \\
(0.000)\end{array}$ & $\begin{array}{l}0.9301 \\
(0.000)\end{array}$ & 0.9350 & $6.65 \mathrm{E}-07$ & $(0.3485)$ & 0.9877 & $(0.3204)$ \\
\hline Noruega & $\begin{array}{c}6.11 \mathrm{E}-06 \\
(0.000)\end{array}$ & $\begin{array}{c}0.0317 \\
(0.0037)\end{array}$ & $\begin{array}{l}0.0714 \\
(0.000)\end{array}$ & $\begin{array}{l}0.9119 \\
(0.000)\end{array}$ & 0.9437 & $9.94 \mathrm{E}-07$ & $(0.2499)$ & 0.0012 & $(0.9722)$ \\
\hline Portugal & $\begin{array}{c}6.38 \mathrm{E}-06 \\
(0.000)\end{array}$ & $\begin{array}{c}0.0296 \\
(0.0111)\end{array}$ & $\begin{array}{l}0.0922 \\
(0.000)\end{array}$ & $\begin{array}{l}0.8573 \\
(0.000)\end{array}$ & 0.8869 & $1.57 \mathrm{E}-05$ & $(0.000)$ & 0.7737 & $(0.3792)$ \\
\hline Suiza & $\begin{array}{c}3.42 \mathrm{E}-06 \\
(0.000)\end{array}$ & $\begin{array}{c}0.0070 \\
(0.4468)\end{array}$ & $\begin{array}{l}0.1101 \\
(0.000)\end{array}$ & $\begin{array}{l}0.9158 \\
(0.000)\end{array}$ & 0.9228 & $5.14 \mathrm{E}-07$ & $(0.2333)$ & 1.1494 & $(0.2838)$ \\
\hline
\end{tabular}


Los resultados reportados sobre la variable dummy indican que la crisis financiera ha incrementado la magnitud del efecto apalancamiento en los mercados financieros de Perú, Grecia, Francia, Alemania, Reino Unido, Italia, España, Irlanda, Argentina y Portugal, la mayoría de ellos europeos y parte de la zona euro. En lo que respecta a los mercados de Colombia, Chile, México, EUA, Canadá, Brasil, Suecia, Noruega y Suiza, el efecto asimetría no incrementó, a raíz de los efectos de la crisis, ya que los coeficientes asociados a la variable dummy presentaron signos negativos o probabilidad mayor a $5 \%$, siendo no significativos estadísticamente.

Por su parte, los valores del estadístico ARCH- LM con un rezago son no significativos estadísticamente, ya que la probabilidad asociada es mayor al 0.05 , rechazando la presencia de efecto ARCH después de la aplicación del modelo, lo cual sugiere que los modelos son adecuados y que proveen buen ajuste.

Los resultados obtenidos a partir de los modelos GARCH simétricos y asimétricos tienen graves implicaciones económicas y financieras para los países latinoamericanos, ya que el incremento en la volatilidady persistencia en la misma, se traducen a los mercados en mayor riesgo sobre los rendimientos de una acción o conjunto de acciones en el largo plazo.

Al aumentar el riesgo los capitales se retiran o piden mayor rendimiento para su permanencia, restringiendo y encareciendo el financiamiento, a través de uno de los principales canales, el bursátil. El encarecimiento y restricción del financiamiento tiene graves implicaciones para las empresas productivas ya que impacta sus costos, competitividad y actividad, afectando variables macro como la inversión, producción y consumo, impactando en un segundo momento a la actividad económica real.

En cuanto a la asimetría, significa que cuando hay caídas en las bolsas de valores se presentamayor variación sobre los rendimientos, es decir mayor riesgo, impactando las expectativas de los inversionistas e incrementando su aversión a invertir, sobre todo en mercados en desarrollo.

\section{Conclusiones}


La presente investigación analiza el impacto de la crisis financiera global en la dinámica de los mercados accionarios más importantes del continente americano y europeo. La metodología empleada para lograr dicho objetivo es la modelación GARCH simétrica y asimétrica con la incorporación de variable dummy en la ecuación de varianza.

Como primer acercamiento, el análisis de correlación aporta indicios sobre cambios en la dinámica de los índices bursátiles en el periodo en el cual se presentan los efectos de la crisis financiera global. Para comprobar si los efectos de la crisis impactaron la dinámica de la volatilidad accionaria, se modelan las series a partir de modelos GARCH simétricos, aportando evidencia de que las series siguen un proceso $\mathrm{ARCH}$ estacionario y que presentan persistencia en su volatilidad. Los resultados de la variable dummy, sugieren que en la mayoría de los mercados bursátiles se incrementó la volatilidad durante el periodo de la crisis.

Los resultados de la modelación GARCH asimétrica sugieren que las series son de carácter asimétrico, las noticias positivas tienen menor impacto que las negativas de la misma magnitud. Igualmente, se apunta a que los mercados de valores europeos fueron aquellos con mayor impacto de la crisis financiera global en su comportamiento, ya que la asimetría se incremento de manera importante en las series a partir de dicha crisis.

Los resultados reportados en el presente estudio tienen aportan información importantepara los inversionistas de cartera, para las empresas que cotizan en dichos mercados y para las autoridades económicas, ya que pueden coadyuvar a una mejor toma de decisiones en cuanto a la relación riesgo-rendimiento y su impacto en la construcción de portafolios internacionales.

Con base en la importancia del tema, la investigación en torno a la volatilidad de los mercados de capital debe seguir desarrollándose. Dentro de las líneas futuras de investigación se podrían realizar estudios en los que se conformen portafolios de inversión por sectores, permitiendo mostrar que, además de un incremento en la volatilidad de los índices y en su asimetría, existen ciertas industrias que se encuentran más afectadas. Alternativamente, se pueden analizar los factores que explican el co-movimiento entre el mercado americano y los países emergentes latinoamericanos y/o europeos. 


\section{Bibliografía}

Acharya, V. V., \& Richardson, M. (2009). Causes of the financial crisis. Critical Review, 21(2-3), 195-210

Apergis, N. (2015). The role of FOMC minutes for US asset prices before and after the 2008 crisis: Evidence from GARCH volatility modeling. The Quarterly Review of Economics and Finance, 55, 100-107.

Babalos, V., Caporale, G. M., \&Spagnolo, N. (2016) Equity Fund Flows and Stock Market Returns in the US before and after the Global Financial Crisis: A VAR-GARCH-inmean Analysis, CESifo Working Paper, No. 5932 https://www.econstor.eu/bitstream/10419/144967/1/cesifo1 wp5932.pdf

Badescu, A., Cui, Z., \& Ortega, J. P. (2015). Non-affine GARCH option pricing models, variance dependent kernels, and diffusion limits. Variance Dependent Kernels, and Diffusion Limits (December 18, 2015).https://ssrn.com/abstract=2705324 or http://dx.doi.org/10.2139/ssrn.2705324

Banulescu, G. D., Hansen, P. R., Huang, Z., \&Matei, M. (2014). Volatility during the financial crisis through the lens of high frequency data: a realized $\mathrm{GARCH}$ approach.http://creates.au.dk/fileadmin/site_files/filer_oekonomi/subsites/creates/Di verse_2015/SoFiE_2015/Papers/129_Volatility_During the_Financial_Crisis_Thro ugh the Lens_of_High_Frequency_Data.pdf

Basher, S. A., \&Sadorsky, P. (2016). Hedging emerging market stock prices with oil, gold, VIX, and bonds: A comparison between DCC, ADCC and GO-GARCH. Energy Economics, 54, 235-247.

Bollerslev, T. (1986). Generalized autoregressive conditional heteroskedasticity. Journal of econometrics, 31(3), 307-327.

Cecchetti, S. G. (2008). Crisis and responses: the Federal Reserve and the financial crisis of 2007-2008 (No. w14134). National Bureau of Economic Research.

Chan, J. C., \& Grant, A. L. (2016). Modeling energy price dynamics: GARCH versus stochastic volatility. Energy Economics, 54, 182-189.

Didier, Love y Martinez (2011) What Explains comovement in stock returns during the 2007-2008 crisis? International Journal of Finance and Economics, 17(2), 182-202.

Dua, P., \&Tuteja, D. (2016). Linkages between Indian and US financial markets: impact of global financial crisis and Eurozone debt crisis.Macroeconomics and Finance in Emerging Market Economies, 1-24.

Greenspan, A. (2008). We will never have a perfect model of risk. Financial Times, 16, 209-23.

Hachula, M., \& Hoffmann, S. (2015). The output effects of commodity price volatility: Evidence from exporting countries (No. 2015/29).http://www.diss.fuberlin.de/docs/servlets/MCRFileNodeServlet/FUDOCS_derivate_000000005543/di scpaper2015_29.pdf?hosts=local

Ismail, M. T., Abdullah, N. A., \& Karim, S. A. A. (2013, April). Impact of global financial crisis on precious metals returns: An application of ARCH and GARCH methods. In AIP Conference Proceedings (Vol. 1522, p. 7).

Joshi, P. (2012). Financial Crisis and Volatility Behaviour of Stock Markets of Asia. QuestJournal of Management and Research, 2(2), 35-44. 
Kim, B. H., Kim, H., \& Lee, B. S. (2015). Spillover effects of the US financial crisis on financial markets in emerging Asian countries. International Review of Economics \& Finance, 39, 192-210.

Klein, T., \& Walther, T. (2015). Oil Price Volatility Forecast with Mixture Memory GARCH. https://www.researchgate.net/publication/273433019_Oil_Price_Volatility Forecast with Mixture Memory GARCH

Kourtis, A., Markellos, R. N., \&Symeonidis, L. (2016). An International Comparison of Implied, Realized, and GARCH Volatility Forecasts. Journal of Futures Markets.

Laeven, L., and F. Valencia, 2012, Systemic Banking Crises Database: An Update. IMF Working 12/163.https://www.imf.org/external/pubs/cat/longres.aspx?sk=26015

Lee, M., Aizenman, J., Jinjarak, Y., \& Park, D. (2012). Developing Countries' Financial Vulnerability to the Euro Crisis: An Event Study of Equity and Bond Markets. http://voxeu.org/article/developing-country-and-emerging-marketvulnerability-eurozone-crisis

Lim, C. M., \&Sek, S. K. (2013). Comparing the performances of GARCH-type models in capturing the stock market volatility in Malaysia. ProcediaEconomics and Finance, 5, 478-487.

López Herrera, F. (2004). Modelado de la volatilidad y pronóstico del índice de precios y cotizaciones de la Bolsa Mexicana de Valores. Contaduría y Administración, (213).

Mishkin, F. S. (2010). Over the cliff: From the subprime to the global financial crisis (No. w16609). National Bureau of Economic Research.http://www.nber.org/papers/w16609

Pilbeam, K., \&Langeland, K. N. (2015). Forecasting exchange rate volatility: GARCH models versus implied volatility forecasts. International Economics and Economic Policy, 12(1), 127-142.

Ping, P. Y., Ahmad, M. H., \& Ismail, N. (2016). Analysis of Volatility Spillover Effects Using Trivariate GARCH Model.http://www.m-hikari.com/ref/ref2016/ref12016/p/ahmadREF1-2016.pdf

Taylor, J. B. (2009). The financial crisis and the policy responses: An empirical analysis of what went wrong (No. w14631). National Bureau of Economic Research.http://www.nber.org/papers/w14631

Teterin, P., Brooks, R., \& Enders, W. (2016). Smooth volatility shifts and spillovers in US crude oil and corn futures markets. Journal of Empirical Finance, 38, 22-36.

Wu, D. D., \& Olson, D. L. (2015). The Real Estate Crash of 2008. In Enterprise Risk Management in Finance (pp. 23-31). Palgrave Macmillan UK.

Zestos, G. K. (2015). The Global Financial Crisis: From US subprime mortgages to European sovereign debt. Routledge. 